\title{
Inventário da Fauna de Hymenoptera Parasitóides Coletados com Redes de Varredura em um Fragmento da Serra da Babilônia, no Sudoeste do Estado de Minas Gerais
}

\author{
Diego Galvão Pádua\& Sônia Lúcia Modesto Zampieron
}

1. Fundação de Ensino Superior de Passos, e-mail: diego.padua.bio@hotmail.com, sonia.zampieron@gmail.com (Autor para correspondência ${ }^{\varpi}$ )

EntomoBrasilis 5 (3): 211-216 (2012)

Resumo. Os himenópteros parasitóides representam o grupo mais rico em espécies dentro da ordem. Este estudo teve por objetivo inventariar a fauna de himenópteros parasitóides, em nível de família, num fragmento da Serra da Babilônia, no sudoeste mineiro, através de redes de varredura, no período de Setembro de 2009 a outubro de 2010. Foram coletados um total de 445 exemplares de himenópteros parasitóides, distribuídos em 20 famílias, contidas em sete superfamílias. Em termos de superfamílias, 55\% eram Chalcidoidea (12 famílias/247 exemplares); 23\% Ichneumonoidea (duas famílias/104 exemplares); 14\% Platygastroidea (duas famílias/64 exemplares); 3\% Proctrotrupoidea (uma família/14 exemplares); 2\% Ceraphronoidea (uma família/sete exemplares); $2 \%$ Cynipoidea (uma família/ sete exemplares); 1\% Chrysidoidea (uma família/ duas exemplares. As superfamílias Chalcidoidea e Ichneumonoidea foram as mais representativas, havendo uma significativa supremacia numérica no primeiro caso: doze famílias ao todo. Destas, duas se sobressaíram em número de exemplares: Eulophidae e Pteromalidae, ambas contendo um grande número de espécies descritas, com hábitos e habitats bastante diversificados e com grande variedade de hospedeiros o que, certamente, pode explicar o grande número de indivíduos coletados. Quanto ao segundo caso, o destaque ficou com a família Braconidae, cujo número de representantes encontrados foi significativamente maior do que a segunda família do grupo. O estudo revelou que a região amostrada ainda se encontra bastante preservada, principalmente no que tange à diversidade de himenópteros parasitóides presentes. Isto garante a manutenção das relações ecológicas e o fortalecimento das cadeias, e fornece os sustentáculos para que o equilíbrio ambiental não se desfaça.

Palavras-Chave: Bioma Cerrado; Hymenoptera parasítica; Parque Nacional Serra da Canastra.

\section{Inventory of the Fauna of Hymenoptera Parasitoids Collected with Sweep Nets in a Fragment of the Serra da Babilonia, in the Southwestern State of Minas Gerais}

Abstract. The hymenopterans parasitoids represent the richest group in species within this order. This study aimed to inventory the fauna of hymenopterans parasitoids, in family level, in a fragment of Serra da Babilônia (Southwest of Minas Gerais), through sweep nets, in a period of September 2009 to October 2010. Were collected a totality of 445 specimens of hymenopterans parasitoids, distributed in 20 families, contained in seven superfamilies. In terms of superfamily, 55\% were Chalcidoidea (12 families/247 specimens); $23 \%$ Ichneumonoidea (two families/104 specimens); $14 \%$ Platygastroidea (two families/64 specimens); $3 \%$ Proctrotrupoidea (one families $/ 14$ specimens); $2 \%$ Ceraphronoidea (one families/ seven specimens); $2 \%$ Cynipoidea (one families/seven specimens); $1 \%$ Chrysidoidea (one families/two specimens). The superfamilies Chalcidoidea and Ichneumonoidea were the most representative, with a significant numerical supremacy in the first case: twelve families in all. These, two excelled in number of specimens: Eulophidae and Pteromalidae, both containing a large number of described species, with habits and habitats very diversified and with large variety of host that certainly can explain the large number of specimens collected. About the second case, the highlight was family Braconidae, that the number of representatives found was significantly higher than the second family of the group. The study realized that the sampled region still is very preserved, mainly in regard to the diversity of hymenopterans parasitoids present. This ensures the maintenance of ecological relationships and the strengthening of chains, and provides the underpinnings for the environmental equilibrium will not come apart.

Keywords: Cerrado Biome; National Park Serra da Canastra; Parasitic Hymenoptera.

A tualmente, um dos principais problemas enfrentados e, consequentemente, um assunto de muito interesse em todo o mundo é a perda da diversidade biológica pela degradação de ambientes, e a respectiva destruição de suas populações naturais. Isto torna necessária a identificação e o registro dos organismos presentes nestas áreas, o mais rápido possível (LANDAU et al. 1999).

Os parasitóides representam o grupo mais comum de inimigos naturais da Classe Insecta, com predominância de espécies de Hymenoptera e, em menor escala, de Diptera (VAn Driesche \& BeLLOWS 1996).

Os Hymenoptera são extremamente abundantes na natureza e ocupam os mais diversos tipos de ambiente disponíveis.
Atualmente estão incluídas nesta ordem cerca de 115.000 espécies, mas estima-se que existam, pelo menos, 250.000 espécies no mundo (Hanson \& GAULD 1995).

Segundo GodFray (1994), são considerados himenópteros parasitóides aquelas espécies cujas larvas se desenvolvem no corpo de outro artrópode, usualmente um inseto, ou em uma massa única ou gregária de hospedeiros, como ootecas ou massas de larvas galhadoras, acarretando a morte do hospedeiro ao final do seu desenvolvimento.

O Cerrado é o segundo maior domínio brasileiro; menor apenas que a Floresta Amazônica. Embora em 1999 ele tenha entrado para a lista dos "hotspots", ou seja, áreas de enorme biodiversidade do mundo e grandemente ameaçadas, nos últimos 
40 anos tem havido uma destruição intensa desse ambiente para expandir fronteiras agrícolas. CoutinHo 2002 enfatiza que aproximadamente $35 \%$ da área deste domínio brasileiro já foram transformadas em pastagens cultivadas e as poucas unidades de conservação encontram-se em situações de completo abandono.

A Serra da Babilônia forma, juntamente com a Serra da Canastra, o Complexo Canastra, uma área entrecortada por cânions, cachoeiras e algumas manchas de Mata Atlântica. Essa Serra, deslumbrante por suas paisagens e mirantes, oferece uma vista privilegiada do paredão da Canastra e da Cachoeira Casca D'Anta. Trata-se de uma área do Parque Nacional da Serra da Canastra ainda não regulamentada e, por isto, cercada de pequenas e médias propriedades rurais sofrendo, inclusive, toda a sorte de degradação antrópica comuns nestas situações.

O difícil acesso à área tem impedido, ou pelo menos dificultado que estudos sejam elaborados no local que, sistematicamente, vem se deteriorando pelas queimadas e desmatamentos, comprometendo todo o patrimônio genético, ecológico e ambiental ali existente.

Diante disso, este trabalho teve como objetivo contribuir para o conhecimento da himenopterofauna parasitóide presente nessa área de Cerrado, mais precisamente na base da Serra da Babilônia, localizada no Município de Delfinópolis, no Sudoeste Mineiro.

\section{MATERIAL E MÉTODOS}

Descrição da área de estudo. A Serra da Babilônia localizase no município de Delfinópolis, sudoeste de Minas Gerais $\left(20^{\circ} 27^{\prime} 47.0\right.$ "S e $\left.046^{\circ} 30^{\prime} 42.7^{\prime \prime} \mathrm{W}\right)$, atingindo $920 \mathrm{~m}$ de altitude e distando $28 \mathrm{~km}$ da entrada principal do Parque Nacional da Serra da Canastra.

A base da Serra, onde ocorreram as coletas com rede de varredura, localiza-se a $908 \mathrm{~m}$ de altitude, com grande influencia antrópica, e possuindo várias fitofisionomias, como campo rupestre, campo limpo, cerrado stricto sensu e matas de galerias (Figura 1).

Nos locais acima de $1000 \mathrm{~m}$, o clima corresponde ao Cwb de Köppen. A temperatura média anual varia de $18^{\circ} \mathrm{C}$ a $20^{\circ} \mathrm{C}$. No mês mais frio, a temperatura média varia de $14^{\circ} \mathrm{C}$ a $16^{\circ} \mathrm{C}$ e a do mês mais quente, varia de $19^{\circ} \mathrm{C}$ a $21^{\circ} \mathrm{C}$. A precipitação média anual varia entre 1400 e $1800 \mathrm{~mm}$, com estação chuvosa no verão e inverno seco.

Em locais abaixo de $1000 \mathrm{~m}$, o clima é o Cwa de Köppen. A temperatura média anual varia de $20^{\circ} \mathrm{C}$ a $23^{\circ} \mathrm{C}$, porém, no mês mais frio varia de $17^{\circ} \mathrm{C}$ a $19,5^{\circ} \mathrm{C}$, e no mês mais quente varia de $21,5^{\circ} \mathrm{C}$ a $24,5^{\circ} \mathrm{C}$. A precipitação média anual é de 1300 a 1800 $\mathrm{mm}$ (IBDF 1981).

Coleta e identificação do material amostrado. O estudo ocorreu de setembro de 2009 a outubro de 2010, perfazendo um total de oito amostras de varreduras, com duração de uma hora, em intervalos regulares de 45 dias.

A rede de varredura usada neste projeto consistia em uma borda em forma de triângulo isósceles, com $36 \mathrm{~cm}$ de comprimento de lado, acoplado a um tecido mais resistente, ovoile. Estetipo de rede é utilizado para insetos que vivem, principalmente, associados à vegetação rasteira, e deve ser utilizada de forma a "varrer" toda a fauna de insetos que se encontra nesta vegetação.

A cada coleta, os insetos capturados foram cuidadosamente transferidos para sacos letais contendo chumaços de algodão embebidos em acetato de etila, de forma que não houvesse perda de nenhum material.

Logo após as coletas no campo, os insetos eram levados para o Laboratório de Entomologia do Curso de Ciências Biológicas da FESP/UEMG, onde eram triados e separados, selecionando os insetos da série parasítica da ordem Hymenoptera, os quais eram inicialmente identificados em nível de superfamílias e, posteriormente, em nível de famílias. As identificações eram feitas com auxílio de um Microscópio estereoscópico e chaves de identificação segundo HANSON \& GAULD (2006).

\section{RESULTADOS E DISCUSSÕES}

Foi coletado um total de 445 exemplares de himenópteros parasitóides, distribuídos em 20 Famílias, contidas em 07 superfamílias, assim representadas: $55 \%$ Chalcidoidea (12 famílias/247 exemplares); 23\% Ichneumonoidea (duas famílias/104 exemplares); 14\% Platygastroidea (duas famílias/64 exemplares); 3\% Proctrotrupoidea (uma família/14 exemplares);

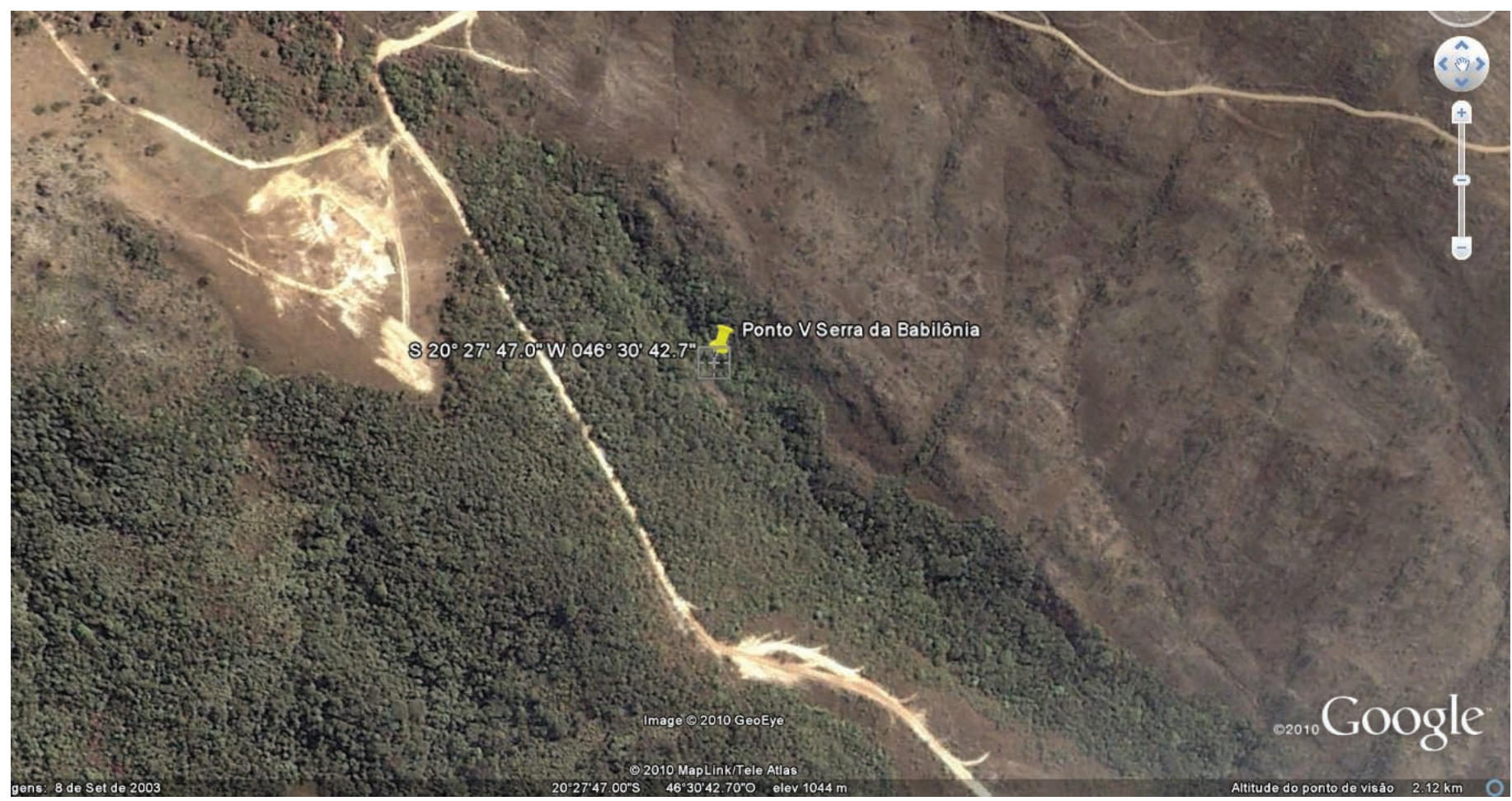

Figura 1. Localização do ponto de coleta dos insetos, utilizando rede de varredura. (Fonte: Google Earth). 
2\% Ceraphronoidea (uma família/ sete exemplares); 2\% Cynipoidea (uma família/ sete exemplares); $1 \%$ Chrysidoidea (uma família/dois exemplares) (Figura 2 e Tabela 1).

Tabela 1. Total de indivíduos de cada superfamília, distribuídos em suas respectivas famílias, coletados entre setembro de 2009 a outubro de 2010, num fragmento da Serra da Babilônia, no Sudoeste do Estado de Minas Gerais.

\begin{tabular}{|c|c|c|}
\hline $\begin{array}{c}\text { Superfamília/ } \\
\text { Família }\end{array}$ & $\mathrm{N}^{\circ}$ de indivíduos & Porcentagem (\%) \\
\hline Chalcidoidea & 247 & 55 \\
\hline Pteromalidae & 83 & \\
\hline Torymidae & 2 & \\
\hline Encyrtidae & 32 & \\
\hline Eurytomidae & 7 & \\
\hline Aphelinidae & 2 & \\
\hline Eulophidae & 110 & \\
\hline Chalcididae & 3 & \\
\hline Eucharitidae & 2 & \\
\hline Signiphoridae & 2 & \\
\hline Mymaridae & 2 & \\
\hline Eupelmidae & 1 & \\
\hline Trichogrammatidae & 1 & \\
\hline Ceraphronoidea & 7 & 2 \\
\hline Ceraphronidae & 7 & \\
\hline Chrysidoidea & 2 & $\mathbf{1}$ \\
\hline Bethylidae & 2 & \\
\hline Platygastroidea & 64 & 14 \\
\hline Platygastridae & 33 & \\
\hline Scelionidae & 31 & \\
\hline Cynipoidea & 7 & 2 \\
\hline Figitidae & 7 & \\
\hline Ichneumonoidea & 104 & $\mathbf{2 3}$ \\
\hline Braconidae & 90 & \\
\hline Ichneumonidae & 14 & \\
\hline Proctotrupoidea & 14 & 3 \\
\hline Diapriidae & 14 & \\
\hline $\begin{array}{c}\text { Total de } \\
\text { Indivíduos } \\
\end{array}$ & 445 & 100 \\
\hline
\end{tabular}

Por esta Tabela pode-se verificar que das superfamílias mais representativas - Chalcidoidea e Ichneumonoidea, a primeira apresentou doze famílias, das quais duas se sobressaíram sobre as demais, Eulophidae e Pteromalidae, com 110 e 83 indivíduos, respectivamente; e no segundo caso, houve uma nítida supremacia numérica da família Braconidae. Estas famílias são constituídas de um número significativo de espécies conhecidas.

A Figura 3 ilustra o número total de famílias de himenópteros parasitóides, por superfamília amostrada.

A família Eulophidae apresenta cerca de 297 gêneros e 4.472 espécies descritas, tanto para áreas tropicais, quanto para temperadas, com ampla variedade de hábitos. Por exemplo, algumas espécies são endo ou ectoparasitoides, idiobiontes ou cenobiontes. Podem ser solitários ou gregários, primários ou hiperparasitóides, especialistas ou generalistas e muitas espécies têm sido estudadas e usadas com sucesso em programas de controle biológico (GAUTHIER et al. 2000).

Além disso, a família supracitada contém espécies conhecidas por atacar ácaros, ovos de aranha, alguns Homoptera, Thysanoptera e um grande número de Coleoptera, Lepidoptera, Diptera e Hymenoptera podendo, inclusive, parasitarem insetos minadores de folhas (Lepidoptera) e comedores de madeira (Coleoptera) (CLAUSEN 1940; AsKEw 1980). Essa enorme variedade de hospedeiros talvez explique a superioridade numérica desta família, em relação aos demais grupos no ambiente estudado.

Quanto à família Pteromalidae, segunda melhor representada, constitui-se de um grande número de espécies parasitóides, muitas das quais com papel importante no controle biológico de muscóides sinantrópicos (RuEda \& Axtell 1985). Contém, aproximadamente, 3100 especies, podendo ser solitárias ou gregárias, ectoparasitóides ou endoparasitóides, parasitóides primários ou secundários e até predadores. A maioria se desenvolve como ectoparasitóides solitários ou gregários em larvas ou pupas de Diptera, Coleoptera, Hymenoptera, Lepidoptera e Siphonaptera (GAULD \& BoLTON 1988).

A supremacia numérica do grupo, apresentada neste estudo, pode estar relacionada a preferência de algumas espécies em parasitar hospedeiros associados à vegetação de pequeno porte, já que as amostras foram obtidas a partir do uso de rede de varredura, cujo raio de ação, normalmente, se estabelece à altura de herbáceas e de pequenos arbustos.

Outro fator que, possivelmente, explique a boa representatividade do grupo nas coletas é o fato de algumas espécies ser encontradas

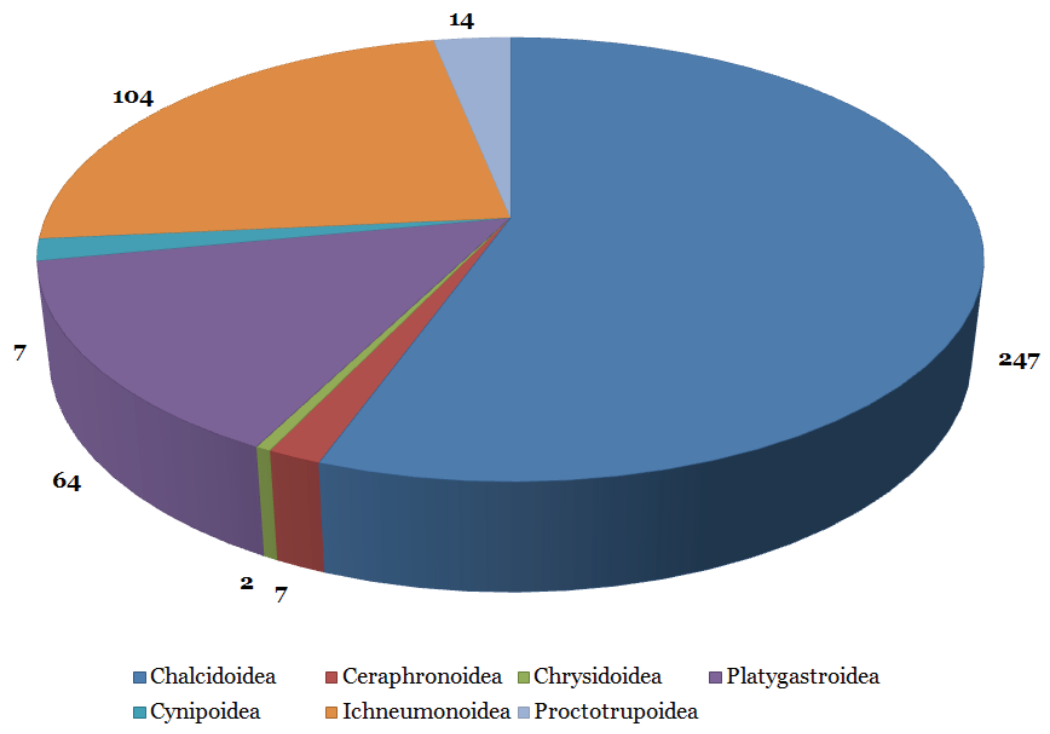

Figura 2. Número total de indivíduos das superfamílias de himenópteros parasitóides amostrados por rede de varredura, coletados entre setembro de 2009 a outubro de 2010, num Fragmento da Serra da Babilônia, no Sudoeste do Estado de Minas Gerais. 


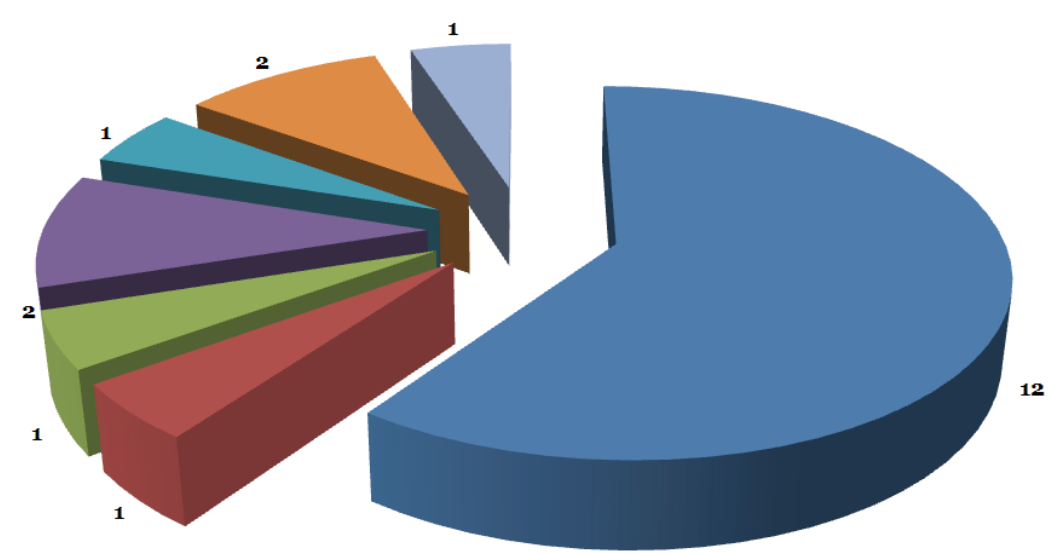

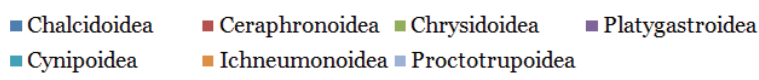

Figura 3. Número total de famílias de himenópteros parasitóides, por superfamília, coletados entre setembro de 2009 a outubro de 2010 , num Fragmento da Serra da Babilônia, no Sudoeste do Estado de Minas Gerais.

associadas à moscas que parasitam frutos. Pteromalidae é uma família bastante conhecida por sua associação com várias famílias de Diptera.

UchôA-Fernandes et al. (2003) pesquisando endoparasitoides larvais de moscas frugívoras da família Tephritidae emergidas de frutos de Cerrado, no Mato Grosso do Sul, obtiveram duas espécies do gênero Spalangia, familia Pteromalidae, o que os levou a concluir que ambas as espécies estavam associadas com pupas dessa mosca.

Segundo Araúso (2011) a densidade populacional de moscasdas-frutas está intimamente relacionada aos seus inimigos naturais. CARvalHo et al. (2000), destacam os parasitóides (Hymenoptera) como os mais efetivos dentre os organismos que atuam no controle biológico natural dessas moscas. As espécies de parasitóide de moscas-das-frutas são pouco específicas. No Brasil pertencem, principalmente, às famílias Braconidae, Figitidae e Pteromalidae.

Apesar de limitarem seus hospedeiros às espécies da família Tephritidae, estes parasitóides possuem uma ampla gama de espécies hospedeiras, atacando moscas-das-frutas de diferentes grupos, em diversos frutos hospedeiros (ARAúJo 2011). Neste caso, a preferência parece estar muito mais relacionada ao ambiente, do que propriamente a esta ou aquela espécie de mosca que ataca frutos.

CALDAS \& Passos (1996) comentando sobre as interações plantaherbívoros e parasitóides, em duas áreas de Cerrado, no sudeste brasileiro, observaram que embora a similaridade de hospedeiros entre as duas áreas tenha sido baixa, a similaridade entre os parasitóides foi alta, sugerindo que os parasitóides têm estratégias específicas para cada situação e que, provavelmente, o parasitismo generalista seja um dos mais importantes, uma vez que permite que os parasitóides usem uma ampla variedade de hospedeiros na ausência de sua espécie favorita, corroborando as conclusões de Araúso (2011).

Dentre os representantes da superfamília Ichneumonoidea, a familia Braconidae foi a que se destacou em número de representantes, em relação à outra família que compõe o grupo. Foram identificados 90 indivíduos da família, dentre os 104 representantes da citada superfamília.

Trata-se da segunda maior família de himenópteros, representada por 34 subfamílias no Novo Mundo (WHARTON et al. 1997). Esta família é representada por vespas parasitóides, cuja influência na dinâmica populacional de alguns grupos de insetos, como lepidópteros e dípteros, tem recebido muita atenção, como os estudos que têm demonstrado o uso eficiente deste grupo em programas de controle biológico (WHARTON 1993). Afinal, a fauna de parasitóides de moscas-das-frutas pertence, principalmente, a essa família.

Considerando que o Cerrado é um bioma definido como "hotspot" de biodiversidade, ou seja, é uma área considerada de alto grau de diversidade biológica em termos de endemismo e que sofre alto grau de ameaça (INTERNATIONAL CONSERVATION 1999 apud MonTeiro 2000), a presença do grande número de representantes desta família pode estar associada a este fato, uma vez que a área estudada possui exatamente estas características, ou seja, trata-se de um fragmento de Cerrado relativamente preservado, constituindo-se num habitat ideal para o desenvolvimento das relações tritróficas constituídas por planta-hospedeiro-parasitóide.

Além disso, o Cerrado apresenta xeromorfia característica o que pode estar favorecendo o estabelecimento de comunidades de Braconidae, cujos hospedeiros ocupam nichos ocultos em tecidos vegetais, sejam eles Lepidoptera, Coleoptera ou Diptera, e de ocorrência pouco comum.

A flora do Cerrado, dada sua diversidade, sem dúvida, contribui na atração de uma grande quantidade de hospedeiros, que por sua vez atraem, principalmente, determinadas famílias de parasitóides, como as melhor representadas nesta pesquisa: Braconidae, Eulophidae e Pteromalidae o que, inclusive, pode ser confirmado na literatura.

Nascimento \& Penteado-Dias (2005) estudando a fauna de hymenoptera associada às espécies de leguminosas que ocorrem em área de Cerrado, observaram que em Stryphnodendron adstringens (Mart.) coville ocorreram espécies de Braconidae (Apanteles sp. 1, Pseudophanerotoma sp. 1), Eulophidae (sp. 1), Pteromalidae (sp. 2), Eupelmidae (sp1), Ichneumonidae (sp. 1), o que foi atribuído ao fato dessas espécies serem parasitóides de outros grupos (Coleoptera e Lepidoptera) que também vivem associados aos frutos do vegetal.

SANTOS et al. (2010) analisando a ocorrência e caracterização de galhas entomógenas em uma área de floresta estacional semidecídua em Goiânia (GO), detectaram-nas em 20 espécies de plantas, de 12 famílias, sendo Leguminosae (nove), Styracaceae (seis) e Ulmaceae (quatro), as que apresentaram o maior número de morfotipos de galhas. Os principais cecidógenos constatados 
foram os dípteros representantes da família Cecidomyiidae, sendo que os parasitóides encontrados pertenciam às famílias Eulophidae, Torymidae, Pteromalidae e Encyrtidae, ou seja, todos pertencentes à superfamília Chalcidoidea (Hymenoptera).

Almeida et al. (2006) estudando a fenologia e artrópodes que ocorrem em Copaifera langsdorffii Desf. também no Cerrado, observaram a emergência dos microhimenópteros parasitóides Brasema sp. (Eupelmidae), Platygaster sp., (Scelionidae), Entedoninae (Eulophidae) das galhas dessa árvore. Para os autores, esse complexo de galhadores, em alta densidade, pode afetar a produção da copaíba, já que ataca diversas partes da planta e, em certos casos, provoca a morte de pontos de crescimento, reduzindo a sua taxa de desenvolvimento, como relatado anteriormente para outras plantas, como a Macairea radula (Bonpl.) (Melastomataceae) (Gonçalves-Alvim et al. 1999).

Diversos autores têm relatado a presença dos gêneros Platygaster e Brasema, bem como outros espécimes da família Eulophidae em galhas de diferentes espécies vegetais, chegando a matar 100\% dos galhadores (AsKew 1980; HAWKINS \& GoEDEN 1984; WEIS \& Abrahamson 1985; Price et al. 1987; Zuparko 1996).

Evidentemente, insetos galhadores estão presentes nos mais diversos ambientes, como pode ser ilustrado pelos estudos de Maia \& Azevedo (2009), que observando micro-himenópteros associados com galhas de Cecidomyiidae (Diptera) em Restingas do Estado do Rio de Janeiro, constataram que Eulophidae, Eupelmidae e Platygastridae foram as famílias frequentes dentre os himenópteros associados com um grande número de famílias de plantas, tais como Myrtaceae, Fabaceae e Malpighiaceae.

Finalmente, o estudo revelou que ainda que hajam áreas já bastante impactadas pelas ações antrópicas, a Serra da Babilônia apresenta uma grande diversidade de himenópteros parasitóides atuando no sentido de regular as populações de hospedeiros comprometidos no ataque aos mais diversos grupos de plantas do Cerrado local. Isto, certamente garante a manutenção das relações ecológicas, o fortalecimento das cadeias e fornece os sustentáculos para que o equilíbrio ambiental não se desfaça.

\section{AGRADECIMENTOS}

Ao Instituto Nacional de Ciência e Tecnologia dos Hymenoptera Parasitóides da Região Sudeste Brasileira (INCT- HYMPAR Sudeste) pela oportunidade e apoio logístico. Ao Conselho Nacional de Pesquisa e Desenvolvimento (CNPq) pela concessão da bolsa de Iniciação científica.

\section{REFERÊNCIAS}

Almeida, C.I.M., G.L.D Leite, S.L. Rocha, M.M.L. Machado \& W.C.H. Maldonado, 2006. Fenologia e artrópodes de Copaifera langsdorffii Desf. no Cerrado. Revista Brasileira Plantas Medicinais, 8: 64-70.

Araújo, A.A.R., 2011. Moscas-das-frutas (Diptera: Tephritidae) e seus parasitóides em frutíferas nativas no estado do Piauí, Brasil. Dissertação (Mestrado em Agronomia) - Universidade Federal do Piauí. 92p.

Askew, R.R., 1980. The diversity of insect communities in leaf mines and plant galls. Journal of Animal Ecology, 49: 81729.

Caldas, A. \& F.C. Passos, 1996. Comments Of Plant-HerbivoreParasitoid Interactions In Two Cerrado Areas of Southern Brazil. Revista Brasileira de Zoologia, 13: 405-410.

Carvalho, R.S., A.S. Nascimento \& W.J. Matrangolo, 2000. Controle biológico. p. 165-179. In: Moscas-das-frutas de importância econômica no Brasil: Conhecimento básico e aplicado. $1^{\text {a }}$ Ed. Ribeirão Preto, Holos. 327p.

Clausen, C.P., 1940, Entomophagous Insects. McGraw-Hill. New York, 688p.

Gauld, I.D. \& B. Bolton, 1988. The Hymenoptera. Oxford
University Press, London, Inglaterra, $331 \mathrm{p}$.

Gauthier, N., J. Lasalle, D.L.J. Quicke \& H.C.J. Godfray, 2000. Phylogeny of Eulophidae (Hymenoptera, Chalcidoidea) with a reclassification of Eulophinae and the recognition that Elasmidae are derived eulophids. Systematic Entomology, 25: 521-539.

Godfray, H.C.J., 1994. Parasitoids, Behavioral and Evolutionary Ecology. Princeton University Press, Princeton. New Jersey. $473 \mathrm{p}$.

Gonçalves-Alvim, S.J., E.C. Landau, M. Fagundes, V.G. Silva, Y. R.F. Nunes \& G.W. Fernandes, 1999. Abundance and impact of a lepidopteran gall on Macairea sp. (Melastomataceae) in the Neotropics. International Journal of Ecology and Environmental Sciences, 25: 115-125.

Hawkins, B.A. \& R.D. Goeden, 1984. Organization of a parasitoid community with a complex of galls on Atriplex spp., in southern California. Ecological Entomology, 9: 271-92.

Hanson, P. E. \& I. D. Gauld, 1995. The Hymenoptera of Costa Rica. Oxford: Oxford University Press, 893 p.

Hanson, P.Y. \& I.D. Gauld, 2006. Hymenoptera de la Región Neotropical. Memories of the American Entomological Institute, 994p.

IBDF. 1981. Plano de Manejo. Parque Nacional da Serra da Canastra. Brasília, Brasiliana Ltda, 81p.

Coutinho, L.M., 2002. O bioma do cerrado. p. 77-92. In: Klein, A.L. (Org.). Eugen Warming e o Cerrado Brasileiro: um século depois. São Paulo, Editora UNESP. 156 p.

Landau, D.; D. Prowell \& C.E. Carlton, 1999. Intensive versus longterm sampling to assess lepidopteran diversity in a southern mixed mesophytic forest. Annals of the Entomological Society of America, 92: 435-441.

Maia, V. C.; M. A.P. Azevedo, 2009. Micro-himenópteros associados com galhas de Cecidomyiidae (Diptera) em Restingas do Estado do Rio de Janeiro (Brasil). Biota Neotropica, 9: 151- 164.

Monteiro, I.S.L., 2000. A abordagem ecossistêmica aplicada ao licenciamento de vegetação natural: o caso do município de Descalvado, SP. Tese de Doutorado. São Paulo, Universidade de São Paulo, 103 p.

Nascimento, A.R. \& A.M. Penteado-Dias, 2005. A fauna de hymenoptera associada às espécies de leguminosas que ocorrem em área de Cerrado. Arquivo do Instituto Biológico de São Paulo, 72: 1-64.

Price, P.W., G.W. Fernandes \& G.L.Waring, 1987. Adaptive nature of insect galls. Environmental Entomology, 16: 15 - 24.

Rueda, L.M. \& R.C. Axtell, 1985. Guide to common species of pupal parasites (Hymenoptera:Pteromalidae) of the house fly and other muscoid flies associated with poultry and livestock manure. Raleigh: North Carolina State University, 88p.

Santos, B.B., H.D. Ferreira \& W.S. Araújo, 2010. Ocorrência e caracterização de galhas entomógenas em uma área de floresta estacional semidecídua em Goiânia, Goiás, Brasil. Acta Botanica Brasilica, 24: 243-249.

Uchôa-Fernandes, M.A, R.M. Molina, I. Oliveira, R.A. Zucchi, N.A. Canal \& N.B. Diaz, 2003. Larval endoparasitoids (Hymenoptera) of frugivorous flies (Diptera, Tephritoidea) reared from fruits of the Cerrado of the State of Mato Grosso do Sul, Brazil. Revista Brasileira de Entomologia. 47: 181186.

Van Driesche, R.G.V \& T.S. Bellows, 1996. Biological control. New York: Chapman \& Hall, 536p.

Weis, A.E. \& W.G. Abrahamson, 1985. Potential selective pressures by parasitoids on plant-herbivore interaction. Ecology, 66: 1261-1269.

Wharton, R.L. 1993. Revisão do Hormiini (Hymenoptera: Braconidae). Com uma descrição de novos táxons. Journal of Natural History, 27: 107-171.

Wharton, R.A., P.M. Marsh \& M.J. Sharkey (Eds), 1997. Manual of the New World Genera of the family Braconidae (Hymenoptera). Special Publication of the International Society of Hymenopterist. 439 p. 
Zuparko, R.L., 1996. Hymenoptera reared from Plagiotrochus suberi (Hymenoptera: Cynipidae) galls in California. PanPacific Entomologist, 72: 27-30
Recebido em: $X X / X X / X X X X$

Aceito em: $X X / X X / X X X X$

Como citar este artigo:

Pádua, D.G. \& S.L.M. Zampieron, 2012. Inventário da Fauna de Hymenoptera Parasitóides Coletados com Redes de Varredura em um Fragmento da Serra da Babilônia, no Sudoeste do Estado de Minas Gerais. EntomoBrasilis, 5(3): 211-216.

Acessível em: http://www.periodico.ebras.bio.br/ojs/index.php/ebras/article/view/221
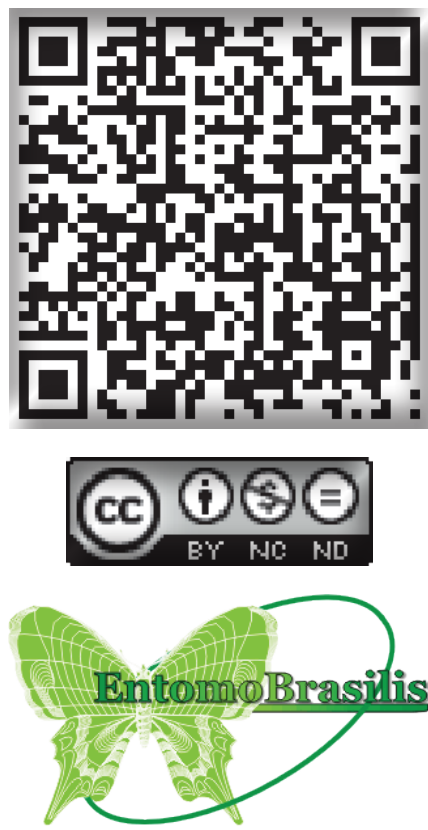